\title{
Thermal Effects in Stokes' Second Problem for Unsteady Second Grade Fluid Flow through a Porous Medium under the Effect Of A Magnetic Field
}

\author{
K. Srinivasa Rao ${ }^{1}$, B. Rama Bhupal Reddy ${ }^{2}$, P. Koteswara Rao ${ }^{3}$ \\ ${ }^{I}$ Research Scholar, Department of Mathematics, Acharya Nagarjuna University, Nagarjuna Nagar, \\ Guntur-522510, A.P., India. \\ ${ }^{2}$ Associate Professor, Dept. of Mathematics, K.S.R.M. College of Engineering, Kadapa, A.P., India. \\ ${ }^{3}$ Professor, Department of Mathematics, Acharya Nagarjuna University, Nagarjuna Nagar, \\ Guntur-522510, A.P., India.
}

\begin{abstract}
In this paper, we investigated the effects of magnetic field and thermal in Stokes' second problem for unsteady second grade fluid flow through a porous medium. The expressions for the velocity field and the temperature field are obtained analytically. The effects of various pertinent parameters on the velocity field and temperature field are studied through graphs in detail.
\end{abstract}

Keywords: Thermal Effects, Fluid Flow, Porous Medium, Magnetic field.

Submitted Date 22 June 2013

Accepted Date: 27 June 2013

\section{Introduction}

The study of non-Newtonian fluid flows past an oscillatory plate has attracted much attention in recent years because of their practical applications. With the growing importance of non-Newtonian fluids in modern technology and industries, investigations of such fluids are desirable. A number of industrially important fluids including molten plastics, polymers, pulps, foods and fossil fuels, which may saturate in underground beds are exhibits non-Newtonian behavior. Due to complexity of fluids, several non-Newtonian fluid models have been proposed. In the category of such fluids, second grade fluid is the simplest subclass for which one can hope to gain an analytic solution. Exact analytic solutions for the flows of non-Newtonian fluids are most welcome provided they correspond to physically realistic situations, as they serve a dual purpose. First, they provide a solution to flow that has technical relevance. Second, such solutions can be used as checks against complicated numerical codes that have been developed for much more complex flows. Various studies on the flows of nonNewtonian fluids have been made under different physical aspects. However some recent contributions in the field may be mentioned (Fetecau and Fetecau [11]; Hayat et al. [14]; Chen et al.[6]; Fetecau and Fetecau[12]; Tan and Masuoka [25]).

The flow of a viscous fluid caused by the sinusoidal oscillation of a flat plate is termed as Stokes' second problem by Schliching [23]. Initially, both the plate and fluid are assumed to be at rest. At time $\mathrm{t}=0+$, the plate suddenly starts oscillating with the velocity $U_{0} e^{i \omega t}$. The study of the flow of a viscous fluid over an oscillating plate is not only of fundamental theoretical interest but it also occurs in many applied problems such as acoustic streaming around an oscillating body, an unsteady boundary layer with fluctuations (Tokuda) [26]. Penton [17] have presented a closed-form to the transient component of the solution for the flow of a viscous fluid due to an oscillating plate. Puri and Kythe [18] have discussed an unsteady flow problem which deals with non-classical heat conduction effects and the structure of waves in Stokes' second problem. Much work has been published on the flow of fluid over an oscillating plate for different constitutive models (Erdogan [9]; Zeng and Weinbaum [28]; Puri and Kythe [19]; Asghar et al. [3]; Ai and Vafai [1]; Ibrahem et al. [15]).

The use of electrically conducting fluids under the influence of magnetic fields in various industries has led to a renewed interest in investigating hydromagnetic flow and heat transfer in different geometrices. For example, Sparrow and Cess [24] have studied the effect of a magnetic field on the free convection heat transfer from surface. Buoyancy driven convection in rectangular enclosure with a transverse magnetic field was studied by Garandet et al. [13]. Chamkha [4] have investigated free convection effects on three-dimensional flow over a vertical stretching surface in the presence of a magnetic field. Erdogan [10] have analyzed the unsteady flow of viscous fluid due to an oscillating plane wall by using Laplace transform technique. Vajravelu and Rivera [27] discussed the hydromagnetic flow at an oscillating plate. Recently, Reddappa et al. [21] have investigated the 
non-classical heat conduction effects in Stokes' second problem of a micropolar fluid under the influence of a magnetic field.

There has been an increase in interest in the effect of porous media, because of their extensive practical applications in geophysics, thermal insulation in buildings, petroleum resources, packed-bed reactors and sensible heat-storage beds. Many studies related to non-Newtonian fluids saturated in a porous medium have been carried out. Dharmadhikari and Kale[7] studied experimentally the effect of non-Newtonian fluids in a porous medium. Chen and Chen [5] investigated the free convection flow along a vertical plate embedded in a porous medium. Rees [22] analyzed the effect of inertia on free convection over a horizontal surface embedded in a porous medium. Nakayama [16] investigated the effect of buoyancy-induced flow over a non-isothermal body of arbitrary shape in a fluid-saturated porous medium. A ray-tracing method for evaluating the radiative heat transfer in a porous medium was examined by Argento [2].

\section{Mathematical Formulation}

We consider the one-dimensional unsteady flow of a laminar, incompressible second grade fluid through a porous medium past a vertical flat plate in the $y z$ - plane and occupy the space $x>0$, with $x$-axis in the vertical direction. A uniform magnetic field $B_{0}$ is applied transverse direction to the flow. It is assumed that the transversely applied magnetic field and magnetic Reynolds number are very small and hence the induced magnetic field is negligible. The plate initially at rest and at constant temperature $\theta_{\infty}$ which is the free stream temperature is moved with a velocity $U_{0} e^{i \omega t}$ in its own plane along the z-axis, and its temperature is subjected to a periodic heating of the form $\left(\theta_{w}-\theta_{\infty}\right) e^{i \omega t}$, where $\theta_{w} \neq \theta_{\infty}$ is some constant.

Viscoelastic fluids can be modeled by Rivlin - Ericksen constitutive equation

$$
\mathrm{S}=-p \mathrm{I}+\mu \mathrm{A}_{1}+\alpha_{1} \mathrm{~A}_{2}+\alpha_{2} \mathrm{~A}_{1}^{2}
$$

where $\mathrm{S}$ is the Cauchy stress tensor, $p$ is the scalar pressure, $\mu, \alpha_{1}$ and $\alpha_{2}$ are the material constants, customarily known as the coefficients of viscosity, elasticity and cross - viscosity, respectively. These material constants can be determined from viscometric flows for any real fluid. $A_{1}$ and $A_{2}$ are Rivlin-Ericksen tensors and they denote, respectively, the rate of strain and acceleration. $A_{1}$ and $A_{2}$ are defined by

$$
\mathrm{A}_{1}=\nabla \mathrm{V}+(\nabla \mathrm{V})^{T}
$$

and

$$
\mathrm{A}_{2}=\frac{d \mathrm{~A}_{1}}{d t}+\mathrm{A}_{1}(\nabla \mathrm{V})+(\nabla \mathrm{V})^{T} \mathrm{~A}_{1}
$$

where $d / d t$ is the material time derivative, $\mathrm{V}$ is the velocity field and $\nabla$ gradient operator and ()$^{T}$ transpose operator. The viscoelastic fluids when modeled by Rivlin-Ericksen constitutive equation are termed as second-grade fluids. A detailed account of the characteristics of second - grade fluids is well documented by Dunn and Rajagopal [8]. Rajagopal and Gupta [20] have studied the thermodynamics in the form of dissipative inequality (Clausius -Duhem) and commonly accepted the idea that the specific Helmholtz free energy should be a minimum in equilibrium. From the thermodynamics consideration they assumed

$$
\mu \geq 0, \quad \alpha_{1}>0, \quad \alpha_{1}+\alpha_{2}=0
$$

We seek the velocity field of the form

$$
V=(u(x, t), 0,0)
$$

For this type of flow, equation of continuity is identically satisfied and the balance of linear momentum reduces to the following differential equation

$$
\rho \frac{\partial u}{\partial t}=\mu \frac{\partial^{2} u}{\partial x^{2}}+\alpha_{1} \frac{\partial^{3} u}{\partial x^{2} \partial t}-\sigma B_{0}^{2} u-\frac{\mu}{k} u+\rho g \beta\left(\theta-\theta_{0}\right)
$$

where $\rho$ is the density of the fluid, $g$ is the acceleration due to gravity, $\beta$ is the coefficient of the thermal expansion and $\sigma$ is the electrical conductivity.

The energy equation (MCF model) is given by (Ibrahem et al.)[15] 


$$
\tau \theta_{t t}+\theta_{t}=\frac{\chi}{\rho c_{p}} \theta_{x x}
$$

Introducing the following non dimensional variables

$$
\bar{x}=\frac{u_{0}}{v} x, \bar{u}=\frac{u}{u_{0}}, \bar{t}=\frac{u_{0}^{2}}{v} t, \bar{\theta}=\frac{\theta-\theta_{0}}{\theta_{w}-\theta_{0}}
$$

into the Equations (6) and (7), we get

$$
\begin{aligned}
& \frac{\partial u}{\partial t}=\frac{\partial^{2} u}{\partial x^{2}}+\alpha \frac{\partial^{3} u}{\partial x^{2} \partial t}+G \theta-\left(M^{2}+\frac{1}{D a}\right) u \\
& p \lambda \frac{\partial^{2} \theta}{\partial t^{2}}+p \frac{\partial \theta}{\partial t}=\frac{\partial^{2} \theta}{\partial x^{2}}
\end{aligned}
$$

where $\quad \alpha=\frac{\alpha_{1} u_{0}^{2}}{\mu \nu}, M^{2}=\frac{\sigma B_{0}^{2}}{\rho u_{0}^{2}} v, G=\frac{v g \beta\left(\theta_{w}-\theta_{0}\right)}{u_{0}^{3}}, D a=\frac{k u_{0}^{2}}{v^{2}} p=\frac{v \rho c_{p}}{\psi}, \lambda=\frac{\tau u_{0}^{2}}{v}$.

The corresponding dimensions are boundary conditions are

$$
\begin{array}{ll}
u(0, t)=e^{i \omega t}, & \theta(0, t)=e^{i \omega t} \\
u(\infty, t)=0, & \theta(\infty, t)=0
\end{array}
$$

\section{Solution}

To solve the non-linear system (8) and (9) using the boundary conditions (10), we assume that $u(x, t)=U(x) e^{i \omega t}, \theta(x, t)=\Theta(x) e^{i \omega t}$

Substituting Equation (11) into Equations (8) and (9) and the boundary conditions (10), we get

$$
\begin{aligned}
& \frac{d^{2} U}{d x^{2}}-m^{2} U=-G n \Theta \\
& \frac{d^{2} \Theta}{d x^{2}}+\left(\lambda p \omega^{2}-i \omega p\right) \Theta=0
\end{aligned}
$$

here $m^{2}=\frac{\left(M^{2}+\frac{1}{D a}\right)+\omega^{2} \alpha+i \omega\left(1-\alpha\left(M^{2}+\frac{1}{D a}\right)\right)}{1+\omega^{2} \alpha^{2}}$ and $n=\frac{1-i \omega \alpha}{1+\omega^{2} \alpha^{2}}$.

The boundary conditions are

$$
\begin{aligned}
& U(0)=1, \Theta(0)=1 \\
& U(\infty)=0, \Theta(\infty)=0
\end{aligned}
$$

Solving the equations (12) - (13) using the boundary conditions Equation (14), we obtain

$$
\begin{aligned}
& U=e^{-m x}+\frac{G n}{k^{2}-m^{2}}\left[e^{-m x}-e^{-k x}\right] \\
& \Theta=e^{-k x}
\end{aligned}
$$

where

$$
k=\sqrt{-\lambda p \omega^{2}+i \omega p}=\sqrt{\omega p\left(\frac{\sqrt{\omega^{2} \lambda^{2}+1}-\lambda \omega}{2}\right)}+i \sqrt{\omega p\left(\frac{\sqrt{\omega^{2} \lambda^{2}+1}+\lambda \omega}{2}\right)} .
$$

The final expressions of the velocity field and temperature field are given by

$$
\begin{aligned}
& u=\left(e^{-m x}+\frac{G n}{k^{2}-m^{2}}\left[e^{-m x}-e^{-k x}\right]\right) e^{i \omega t} \\
& \theta=e^{-k x+i \omega t}
\end{aligned}
$$


The rate of heat transfer coefficient in terms of Nusselt number $N u$ at the wall of the plate is given by

$$
N u=-\left.\frac{\partial \theta}{\partial y}\right|_{y=0}=k e^{i \omega t}
$$

\section{Discussion of the Results}

Figures 1 - 14 show the effects of various values of the pertinent parameters $\alpha, G, D a, M, p$ and $\lambda$ on the velocity $(\operatorname{Re} u$ and $|u|)$ and temperature $(\operatorname{Re} \theta$ and $|\theta|)$ profiles.

Figure 1 shows the effect of material parameter $\alpha$ on $\operatorname{Re} u$ for $M=1, D a=0.1, p=1$, $\omega=10, t=0.1, \lambda=0.005$ and $G=5$. It is found that, the $\operatorname{Re} u$ decreases with increasing $\alpha$. The same trend is observed for $|u|$ from Figure 2. Figure 3 depicts the effect of $G$ on $\operatorname{Re} u$ for $M=1$, $D a=0.1, p=1, \omega=10, t=0.1, \lambda=0.005$ and $\alpha=0.01$. It is observed that, the $\operatorname{Re} u$ first increases and then decreases with increasing $G$. The effect of $G$ on $|u|$ for $M=1, D a=0.1, p=1$, $\omega=10, t=0.1, \lambda=0.005$ and $\alpha=0.01$ is depicted in Figure 4. It is noted that, the $|u|$ increases with an increase in $G$. Figure 5 shows the effect of Darcy $D a$ on $\operatorname{Re} u$ for $G=5, p=1, \omega=10$, $t=0.1, \lambda=0.005$ and $\alpha=0.01$. It is found that, the $\operatorname{Re} u$ first increases and then decreases with increasing $D a$.

The effect of $D a$ on $|u|$ for $G=5, p=1, \omega=10, t=0.1, \lambda=0.005$ and $\alpha=0.01$ is shown in Figure 6. It is observed that, the $|u|$ increases with an increase in $D a$. Figure 7 depicts the effect of Hartmann number $M$ on $\operatorname{Re} u$ for $\quad G=5, \quad D a=0.1, \quad p=1, \quad \omega=10$, $t=0.1, \lambda=0.005$ and $\alpha=0.01$. It is found that, the $\operatorname{Re} u$ first decreases and then increases with increasing $M$. The effect of $M$ on $|u|$ for $G=5, D a=0.1, p=1, \omega=10, t=0.1$, $\lambda=0.005$ and $\alpha=0.01$ is depicted in Figure 8. It is observed that, the $|u|$ decreases with an increase in $M$. Figure 9 shows the effect of $p$ on $\operatorname{Re} u$ for $G=5, M=1, \omega=10, t=0.1, \lambda=0.005$ and $\alpha=0.01$. It is found that, the $\operatorname{Re} u$ first decreases and then increasing with increasing $p$. The effect of $p$ on $|u|$ for $G=5, M=1, D a=0.1, \omega=10, t=0.1, \lambda=0.005$ and $\alpha=0.01$ is depicted in Figure 10. It is noted that, the $|u|$ decreases on increasing $p$.

Figure 11 shows the effects of $\lambda$ on $\operatorname{Re} \theta$ for $G=5, D a=0.1, p=1, \omega=10$, $t=0.1, M=1$ and $\alpha=0.01$. It is observed that, the $\operatorname{Re} \theta$ first increases and then decreases with increasing $\lambda$. Figure 12 depicts the effect of $\lambda$ on $|\theta|$ for $G=5, p=1, \omega=10, t=0.1, M=1$ and $\alpha=0.01$. It is noted that, the $|\theta|$ increases with an increase in $\lambda$. The effect of $p$ on $\operatorname{Re} \theta$ for $G=5$, $\lambda=0.005, D a=0.1, \omega=10, t=0.1, M=1$ and $\alpha=0.01$ is depicted in Figure 13. It is found that, the $\operatorname{Re} \theta$ first decreases and then increases with an increase in $p$. Figure 14 illustrates the effect of $p$ on $|\theta|$ for $G=5, D a=0.1, \lambda=0.005, \omega=10, t=0.1, M=1$ and $\alpha=0.01$. It is observed that, the $|\theta|$ decreases with increasing $p$.

Table-1 shows the effect of $p$ on Nusselt number $\operatorname{Re} N u$ with $t=0.1, \lambda=0.005$ and $\omega=1$. It is found that, the $\operatorname{Re} N u$ increases with increasing $p$. Table- 2 depicts the effect of $\omega$ on Nusselt number $\operatorname{Re} N u$ with $t=0.1, \lambda=0.005$ and $p=1$. It is noted that, the $\operatorname{Re} N u$ increases with increasing $\omega$. 


\section{Conclusions}

In this paper, the thermal effect in Stokes second problem for unsteady second grade fluid flow through a porous medium under the influence of magnetic field is investigated. The expressions for the velocity field and the temperature field are obtained analytically. It is found that, the $\operatorname{Re} u$ and $|u|$ decreases with increasing $\alpha, M$ and $p$, while they increases with increasing $G$ and $D a$. The $\operatorname{Re} \theta$ and $|\theta|$ increase with increasing $\lambda$, while they decrease with increasing $p$.

Table-1: Effect of $p$ on Nusselt number $\operatorname{Re} N u$ with $t=0.1, \lambda=0.005$ and $\omega=1$.

\begin{tabular}{|c|c|}
\hline$p$ & $N u$ \\
\hline 0.2 & $\mathbf{0 . 2 8 2 2}$ \\
\hline $\mathbf{0 . 5}$ & $\mathbf{0 . 4 4 6 2}$ \\
\hline $\mathbf{0 . 7}$ & $\mathbf{0 . 5 2 8 0}$ \\
\hline 1 & $\mathbf{0 . 6 3 1 0}$ \\
\hline
\end{tabular}

Table-2: Effect of $\omega$ on Nusselt number $\operatorname{Re} N u$ with $t=0.1, \lambda=0.005$ and $p=1$.

\begin{tabular}{|c|c|}
\hline$\omega$ & $N u$ \\
\hline $\mathbf{0}$ & $\mathbf{0}$ \\
\hline $\mathbf{1}$ & $\mathbf{0 . 6 3 1 0}$ \\
\hline $\mathbf{2}$ & $\mathbf{0 . 7 7 5 5}$ \\
\hline $\mathbf{3}$ & $\mathbf{0 . 7 9 6 6}$ \\
\hline
\end{tabular}

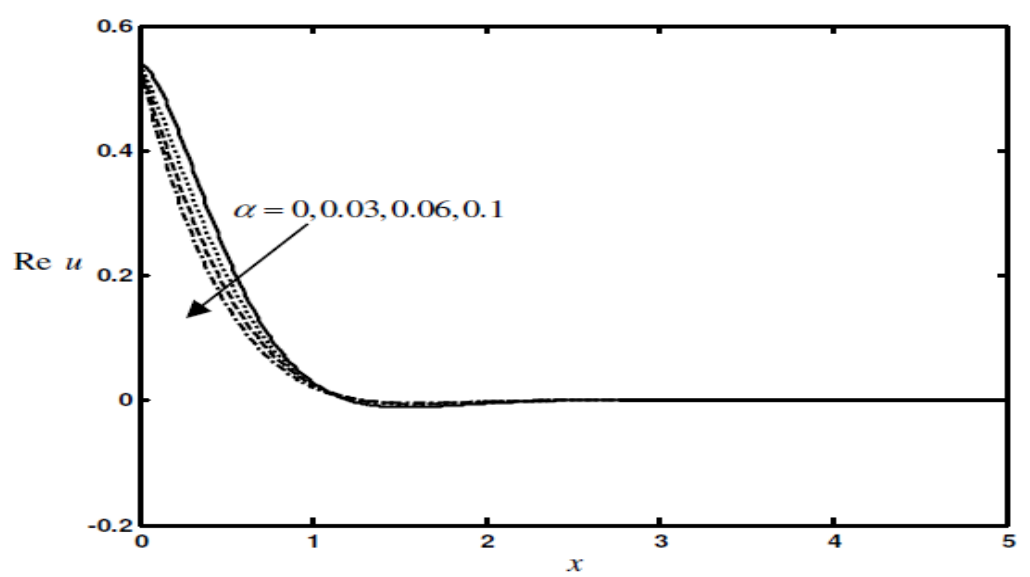

Fig. 1. Effect of $\alpha$ on $\operatorname{Re} u$ for $M=1, p=1, D a=0.1, \omega=10, t=0.1$, $\lambda=0.005$ and $G=5$.

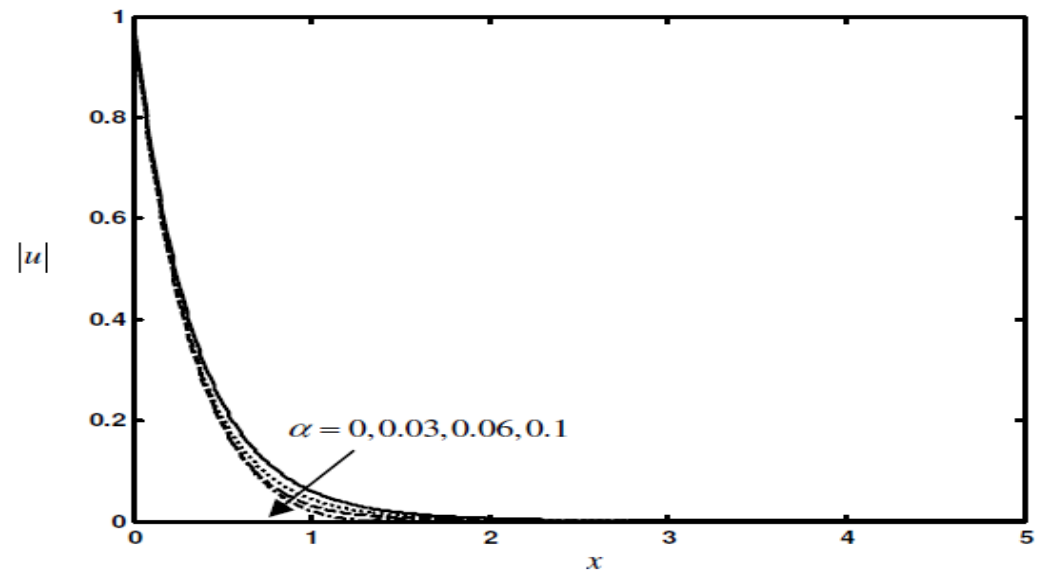

Fig. 2. Effect of $\alpha$ on $|u|$ for $M=1, p=1, \omega=10, t=0.1, D a=0.1$, $\lambda=0.005$ and $G=5$. 


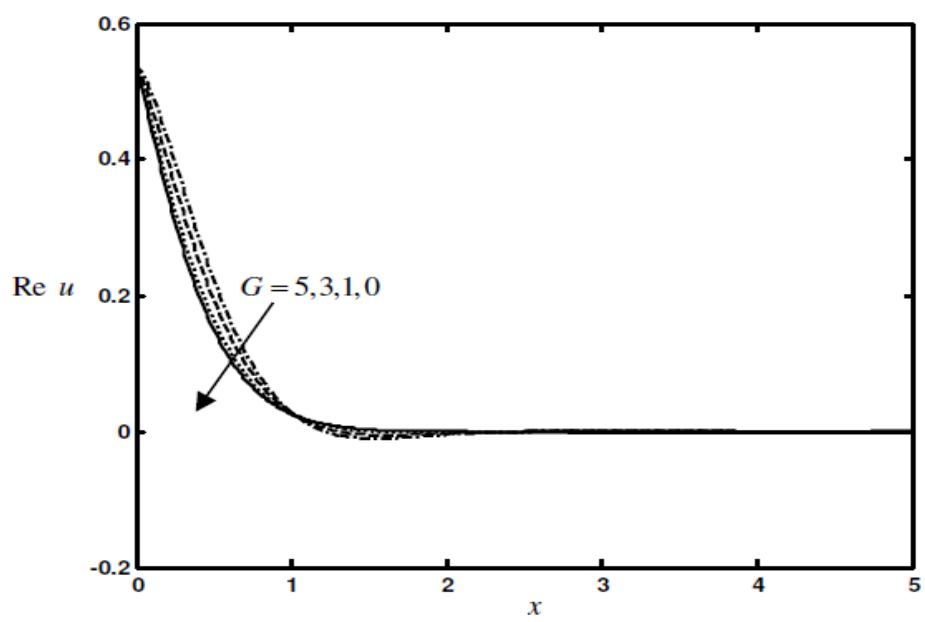

Fig. 3. Effect of $G$ on $\operatorname{Re} u$ for $M=1, p=1, \omega=10, t=0.1, D a=0.1$ $\lambda=0.005$ and $\alpha=0.01$.

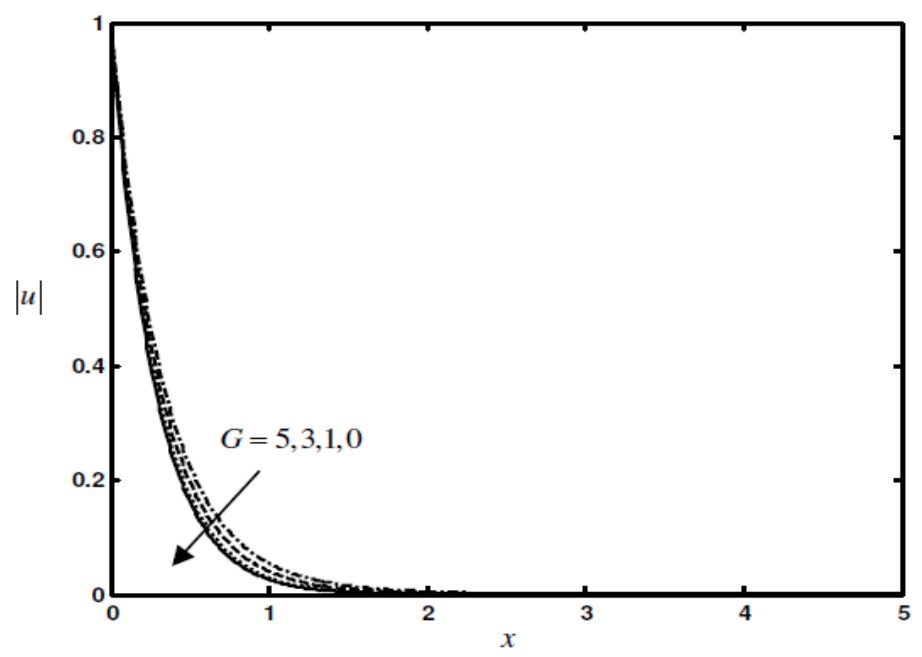

Fig. 4. Effect of $G$ on $|u|$ for $M=1, p=1, \omega=10, t=0.1, D a=0.1$, $\lambda=0.005$ and $\alpha=0.01$.

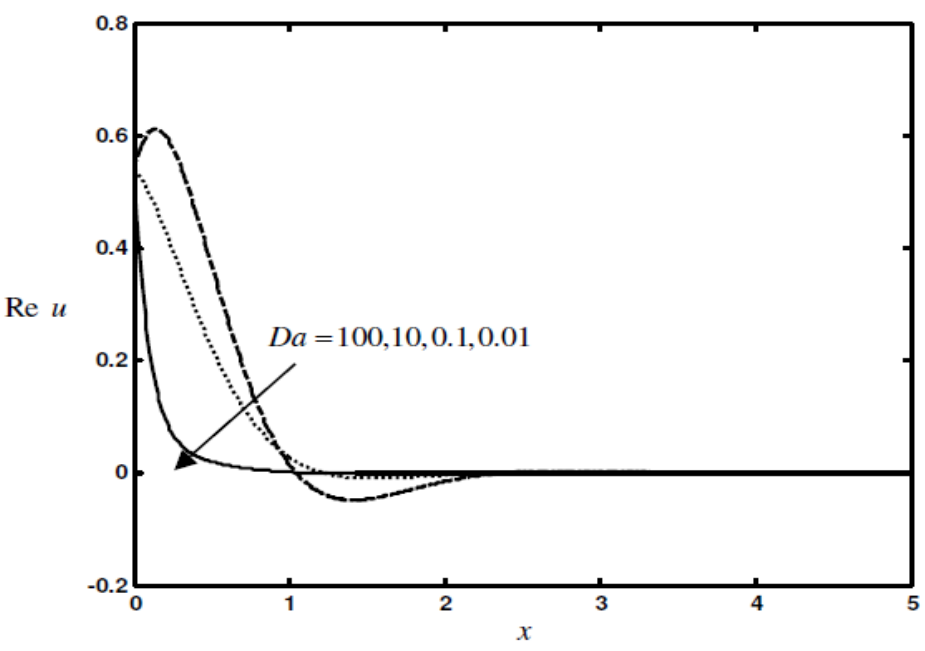

Fig. 5. Effect of $D a$ on $\operatorname{Re} u$ for $G=5, M=1, p=1, \omega=10, t=0.1$, $\lambda=0.005$ and $\alpha=0.01$. 


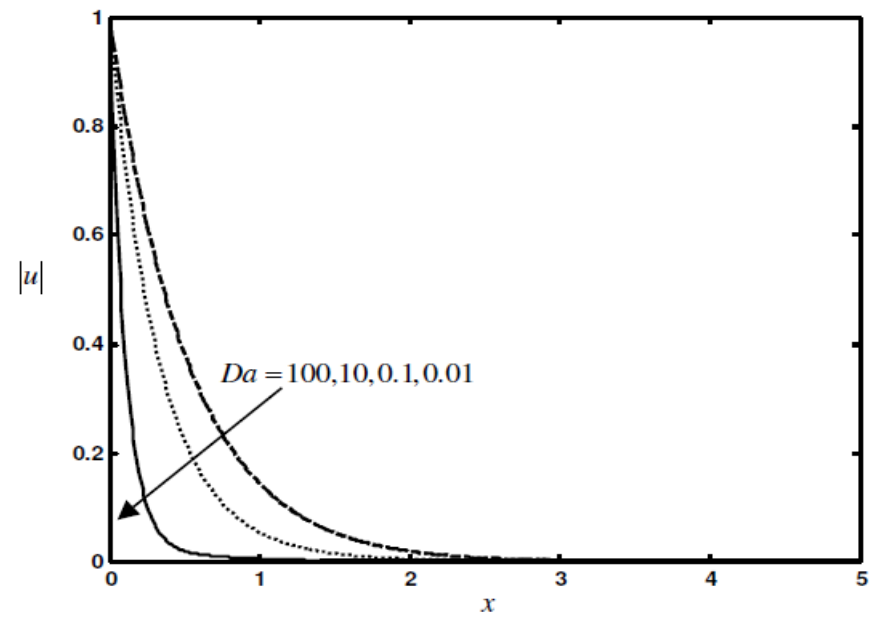

Fig. 6. Effect of $D a$ on $|u|$ for $G=5, p=1, M=1, \omega=10, t=0.1$, $\lambda=0.005$ and $\alpha=0.01$.

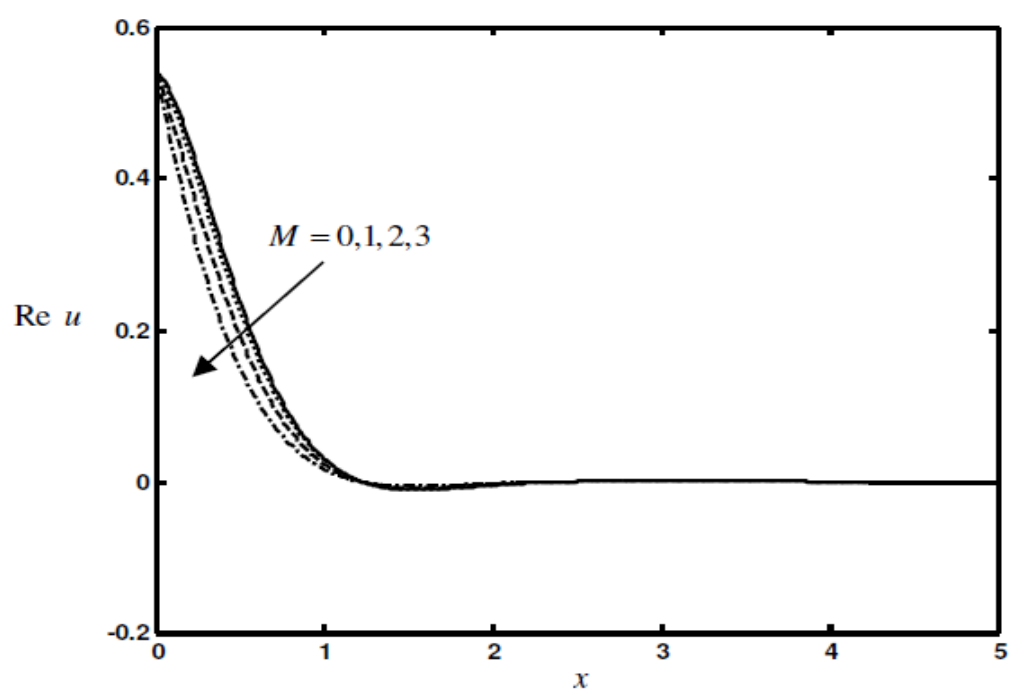

Fig. 7. Effect of $M$ on $\operatorname{Re} u$ for $G=5, p=1, \omega=10, D a=0.1, t=0.1$, $\lambda=0.005$ and $\alpha=0.01$.

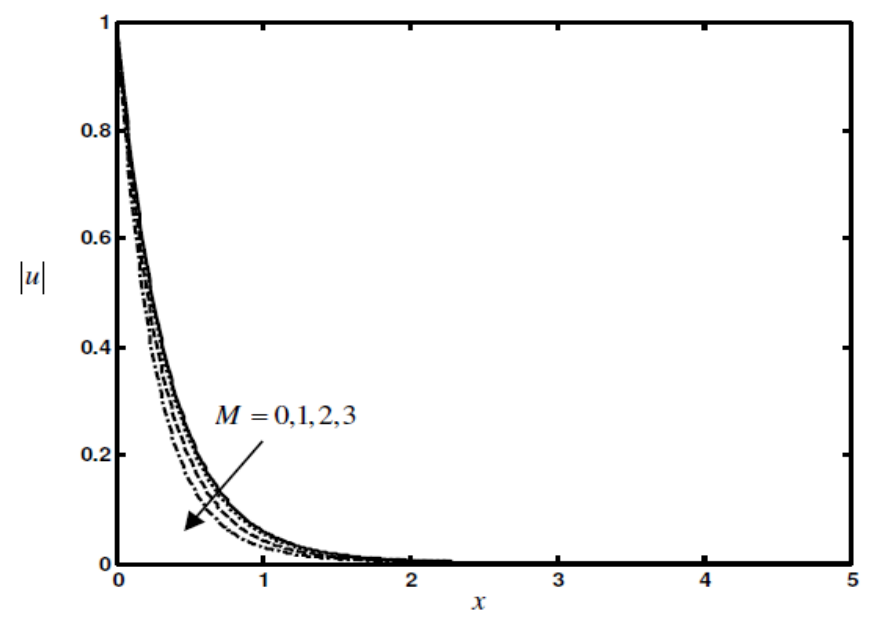

Fig. 8. Effect of $M$ on $|u|$ for $G=5, p=1, \omega=10, t=0.1, D a=0.1$, $\lambda=0.005$ and $\alpha=0.01$. 


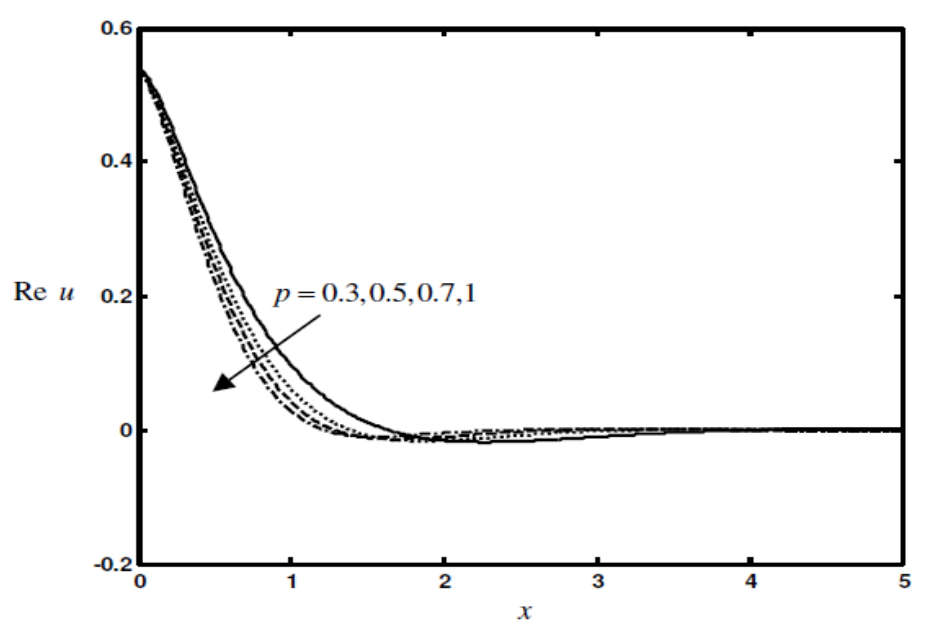

Fig. 9. Effect of $p$ on $\operatorname{Re} u$ for $G=5, M=1, \omega=10, t=0.1, D a=0.1$, $\lambda=0.005$ and $\alpha=0.01$.

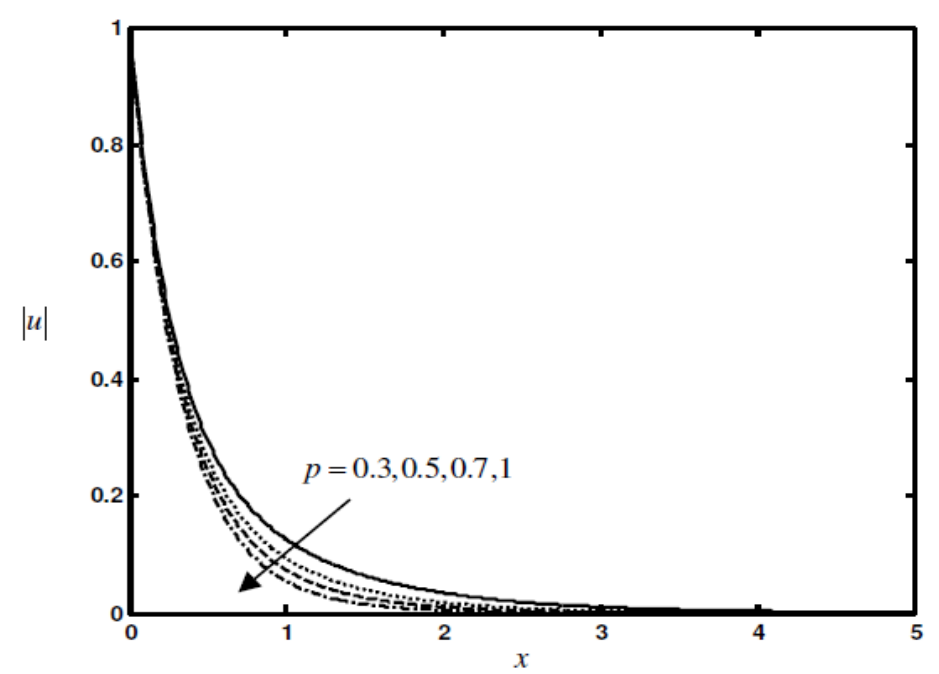

Fig. 10. Effects of $p$ on $|u|$ for $G=5, M=1, \omega=10, D a=0.1, t=0.1$, $\lambda=0.005$ and $\alpha=0.01$.

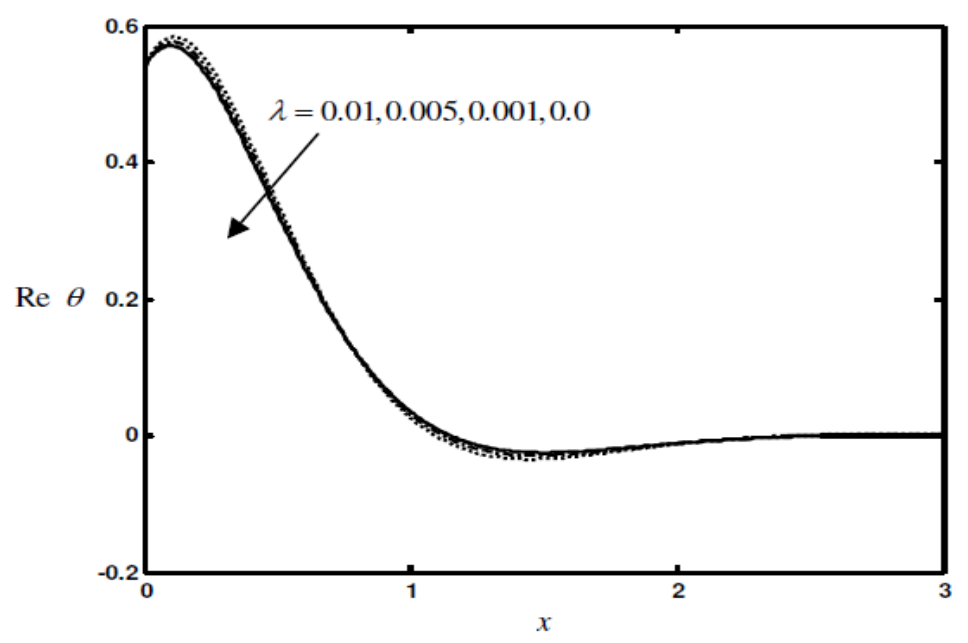

Fig. 11. Effects of $\lambda$ on $\operatorname{Re} \theta$ for $G=5, p=1, \omega=10, D a=0.1, t=0.1$, $M=1$ and $\alpha=0.01$. 


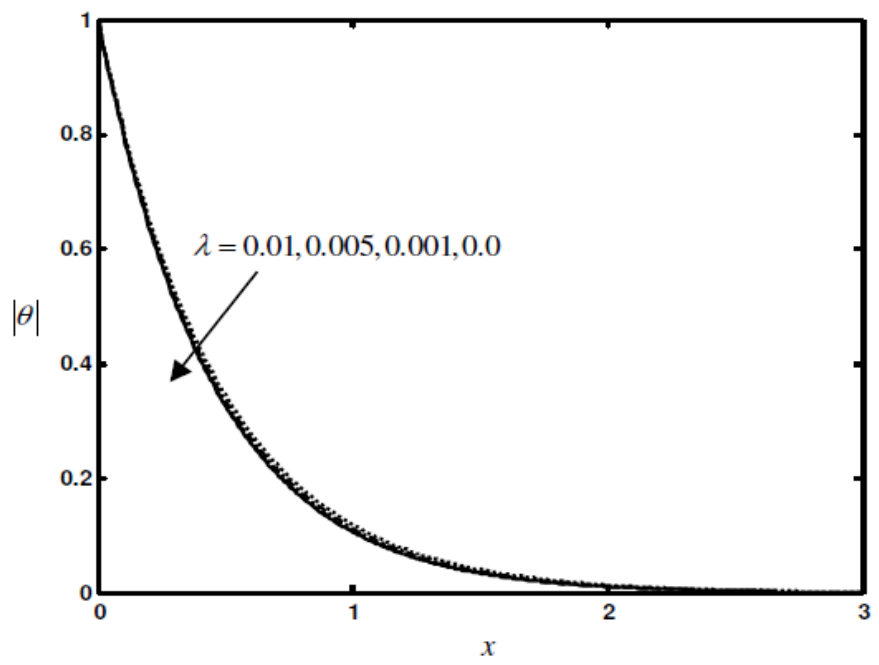

Fig. 12. Effects of $\lambda$ on $|\theta|$ for $G=5, p=1, D a=0.1, \omega=10, t=0.1$, $M=1$ and $\alpha=0.01$.

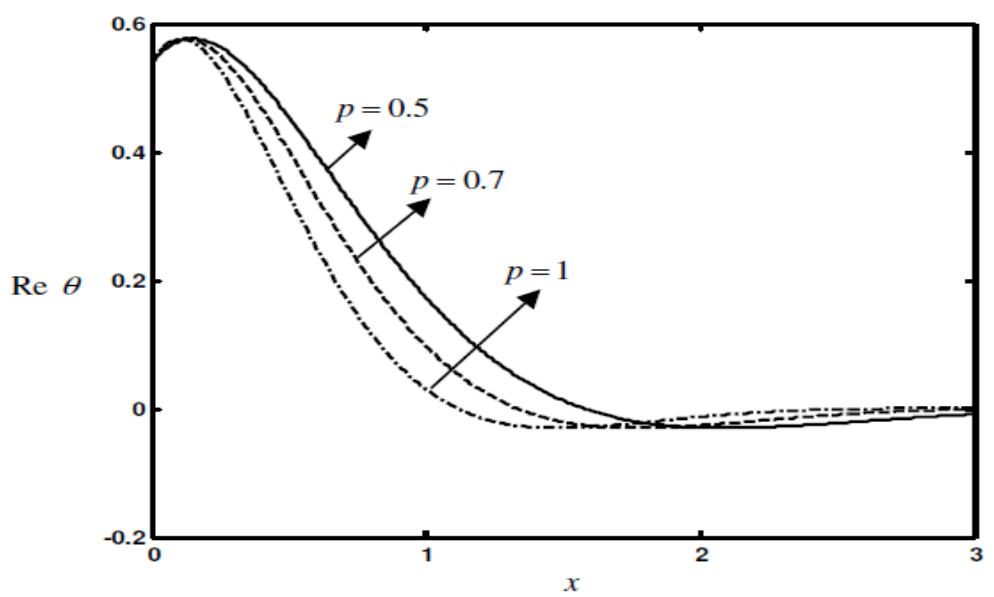

Fig. 13. Effects of $p$ on $\operatorname{Re} \theta$ for $G=5, \lambda=0.005, \omega=10, D a=0.1$, $t=0.1, M=1$ and $\alpha=0.01$.

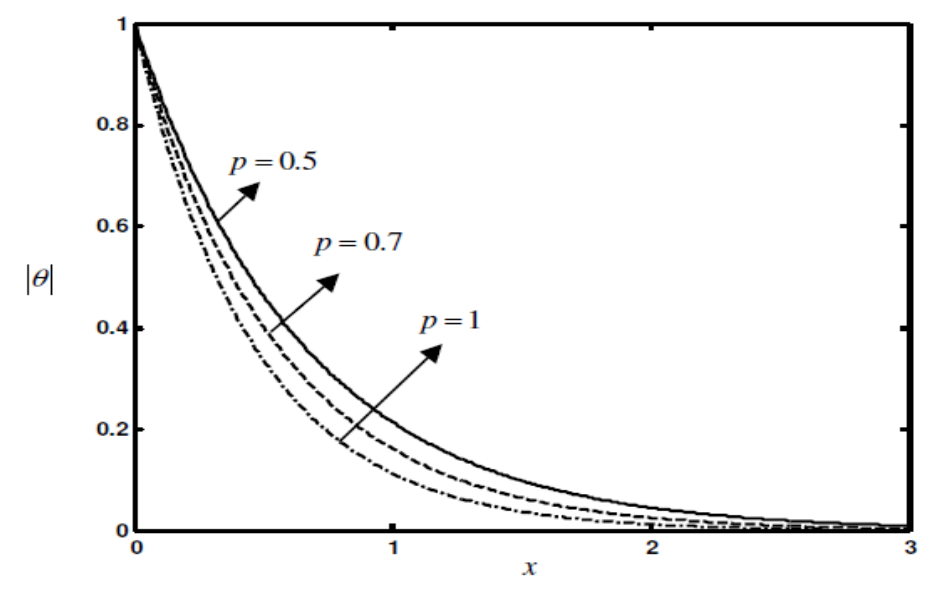

Fig. 14. Effects of $p$ on $|\theta|$ for $G=5, \lambda=0.005, \omega=10, D a=0.1, t=0.1$, $M=1$ and $\alpha=0.01$. 


\section{References}

[1] L. Ai and K. Vafai, An investigation of stokes' second problem for non-Newtonian fluids, Numerical Heat Transfer, Part A, 47(2005), 955-980.

[2] C. Argento and D. Bouvard, A Ray, Tracing Method for Evaluating the Radiative Heat Transfer in Porous Medium", Int. J. Heat Mass Transfer, 39(1996), 3175-3180.

[3] S. Asghar, T. Hayat and A.M. Siddiqui, Moving boundary in a non-Newtonian fluid, Int. J. Nonlinear Mech., $37(2002), 75$ - 80.

[4] A. J. Chamkha, Hydromagnetic three-dimensional free convection on a vertical stretching surface with heat generation or absorption, Int. J. Heat Fluid Flow, 20(1999), 84 - 92.

[5] H. Chen and C. Chen, Free convection flow of non-newtonian fluids along a vertical plate embedded in a porous medium, Journal of Heat Transfer, 110(1988), $257-260$.

[6] C.I. Chen, C.K. Chen and Y.T. Yang, Unsteady unidirectional flow of an Oldroyd-B fluid in a circular duct with different given volume flow rate conditions. Heat and Mass Transfer, 40(2004), 203 - 209.

[7] R.V. Dharmadhikari and D.D. Kale, Flow of non-newtonian fluids through porous media, Chem. Eng. Sci., 40(1985), 527-529.

[8] J.E. Dunn and K.R. Rajagopal, Fluids of differential type: critical review and thermodynamic analysis, Int. J. Engng. Sci., 33(1995), $689-729$.

[9] M. E. Erdogan, Plane surface suddenly set in motion in a non-Newtonian fluid, Acta Mech., 108(1995), 179 - 187.

[10] M. E. Erdogan, A note on an unsteady flow of a viscous fluid due to an oscillating plane wall, Int. J. Non-Linear Mech., $35(2000), 1-6$.

[11] C. Fetecau and C. Fetecau, A new exact solution for the flow of Maxwell fluid past an infinite plate, Int. J. Non-Linear Mech., $38(2003), 423-427$.

[12] C.Fetecau and C.Fetecau, Starting solutions for some unsteady unidirectional flows of a second grade fluid, Int. J. Engng. Sci., 43(2005), $781-789$.

[13] J.P. Garandet, T. Alboussiere and R. Morau, Buoyancy driven convection in a rectangular enclosure with a transverse magnetic field, Int. J. Heat Mass Transfer, 35(1992), $741-749$.

[14] T. Hayat, Y. Wang and K. Hutter, Hall effects on the unsteady hydromagnetic oscillatory flow of a second grade fluid. Int. J. Non-Linear Mech., 39(2004), 1027 - 1037.

[15] F. S. Ibrahim, I. A. Hassanien and A. A. Bakr, Thermal effects in Stokes' second problem for unsteady micropolar fluids flow, Applied Mathematics and Computation, 173(2006), 916 - 937.

[16] A. Nakayama and H. Koyama, Buoyancy-induced flow of non-newtonian fluids over a non-isothermal body of arbitrary shape in a porous medium, Applied Scientific Research, 48(1991), 55-70.

[17] R. Penton, The transient for Stokes' oscillating plane: a solution in terms of tabulated functions, J. Fluid Mech., 31(1968), 819 825 .

[18] P. Puri and P.K. Kythe, Thermal effects in Stokes' second problem, Acta Mech., 112(1998), 44 - 50.

[19] P. Puri and P.K. Kythe, Stokes' first and second problems for Rivlin-Ericksen fluids with non classical heat conduction, ASME J. Heat Transfer, 120(1998), 44 - 50.

[20] K. R. Rajagopal and A. S.Gupta. An exact solution for the flow of a non-Newtonian fluid past an infinite porous plate, Mecanica, 19(1984), $158-160$.

[21] B. Reddappa, M. V. Subba Reddy and K. R. Krishna Prasad,Thermal effects in Stokes' second problem for unsteady magneto hydrodynamic flow of a Micropolar fluid, Vol. 21(3)(2009), 365 - 373.

[22] D.A.S. Rees, The Effect of Inertia on Free Convection from a Horizontal Surface Embedded ina Porous Medium", Int. J. Heat Mass Transfer, 39 (1996), 3425-3430, 1996.

[23] H. Schlichting, K. Gersten, Boundary Layer Theory, 8th edition, Springer, Berlin, 2000.

[24] E.M. Sparrow and R.D. Cess, Effect of magnetic field on free convection heat transfer, Int. J. Heat Mass Transfer, 3(1961), 267 274.

[25] W. C.Tan and T. Masuoka, Stokes first problem for second grade fluid in a porous half space. Int. J. Non-Linear Mech., 40(2005), $515-522$.

[26] N. Tokuda, On the impulsive motion of a flat plate in a viscous fluid, J. Fluid Mech. 33(1968), 657 - 672.

[27] K. Vajravelu and J. Rivera, Hydromagnetic flow at an oscillating plate, Int. J. Non-Linear Mech., $38(2003), 305$ - 312.

[28] Y. Zeng and S. Weinbaum, Stokes' problem for moving half planes, J. Fluid Mech., 287(1995), 59 - 74. 